TRANSACTIONS OF THE

AMERICAN MATHEMATICAL SOCIETY

Volume 362, Number 7, July 2010, Pages 3591-3612

S 0002-9947(10)05014-2

Article electronically published on February 17, 2010

\title{
THE INVERSE INTEGRATING FACTOR AND THE POINCARÉ MAP
}

\author{
ISAAC A. GARCÍA, HÉCTOR GIACOMINI, AND MAITE GRAU
}

Dedicated to Professor Javier Chavarriga

\begin{abstract}
This work is concerned with planar real analytic differential systems with an analytic inverse integrating factor defined in a neighborhood of a regular orbit. We show that the inverse integrating factor defines an ordinary differential equation for the transition map along the orbit. When the regular orbit is a limit cycle, we can determine its associated Poincaré return map in terms of the inverse integrating factor. In particular, we show that the multiplicity of a limit cycle coincides with the vanishing multiplicity of an inverse integrating factor over it. We also apply this result to study the homoclinic loop bifurcation. We only consider homoclinic loops whose critical point is a hyperbolic saddle and whose Poincaré return map is not the identity. A local analysis of the inverse integrating factor in a neighborhood of the saddle allows us to determine the cyclicity of this polycycle in terms of the vanishing multiplicity of an inverse integrating factor over it. Our result also applies in the particular case in which the saddle of the homoclinic loop is linearizable, that is, the case in which a bound for the cyclicity of this graphic cannot be determined through an algebraic method.
\end{abstract}

\section{InTRODUCTION AND STATEMENT OF THE MAIN RESUltS}

We consider two-dimensional autonomous systems of real differential equations of the form

$$
\dot{x}=P(x, y), \quad \dot{y}=Q(x, y),
$$

where $P(x, y)$ and $Q(x, y)$ are analytic functions defined on an open set $\mathcal{U} \subseteq \mathbb{R}^{2}$. Here, the dot denotes, as usual, the derivative with respect to the independent variable $t$. The vector field associated to system (1) will be denoted by $\mathcal{X}=$ $P(x, y) \partial_{x}+Q(x, y) \partial_{y}$ and its divergence is $\operatorname{div} \mathcal{X}:=\partial P / \partial x+\partial Q / \partial y$.

Definition 1. An inverse integrating factor for system (1) in $\mathcal{U}$ is a nonlocally null $\mathcal{C}^{1}$ solution $V: \mathcal{U} \subset \mathbb{R}^{2} \rightarrow \mathbb{R}$ of the linear partial differential equation

$$
\mathcal{X} V=V \operatorname{div} \mathcal{X} .
$$

We remark that if $V$ is an inverse integrating factor of system (10), then we have $\operatorname{div}(\mathcal{X} / V) \equiv 0$ and this is the origin of its name. Moreover, the zero-set of $V$, $V^{-1}(0):=\{(x, y) \mid V(x, y)=0\}$, is composed of trajectories of (10). In fact it is

Received by the editors October 172007 and, in revised form, May 8, 2008.

2010 Mathematics Subject Classification. Primary 37G15, 37G20, 34C05.

Key words and phrases. Inverse integrating factor, Poincaré map, limit cycle, homoclinic loop. The authors were partially supported by a DGICYT grant number MTM2005-06098-C02-02.

(C)2010 American Mathematical Society Reverts to public domain 28 years from publication 
easy to see that for any point $p \in \mathcal{U}$, if $\Phi(t ; p)$ is the orbit of (11) that satisfies $\Phi(0 ; p)=p$, then

$$
V(\Phi(t ; p))=V(p) \exp \left(\int_{0}^{t} \operatorname{div} \mathcal{X} \circ \Phi(s ; p) d s\right) .
$$

Thus if $V(p)=0$, then $V(\Phi(t ; p))=0$ for all $t$ provided that $V$ is defined on $\Phi(t ; p)$.

We consider a certain regular orbit of system (11) and we assume the existence of an analytic inverse integrating factor in a neighborhood of this regular orbit. We show that some qualitative properties of the orbits in a neighborhood of the considered regular orbit can be deduced from the known inverse integrating factor.

We consider a regular orbit $\phi(t)$ of system (11) and two transversal sections $\Sigma_{1}$ and $\Sigma_{2}$ based on it. We are going to study the transition map of the flow of system (11) in a neighborhood of this regular orbit. This transition map is studied by means of the Poincaré map $\Pi: \Sigma_{1} \rightarrow \Sigma_{2}$, which is defined as follows. Given a point in $\Sigma_{1}$, we consider the orbit of system (11) with it as initial point and we follow this orbit until it first intersects $\Sigma_{2}$. The map $\Pi$ makes correspond to the point in $\Sigma_{1}$ the encountered point in $\Sigma_{2}$.

Let $(\varphi(s), \psi(s)) \in \mathcal{U}$, with $s \in \mathcal{I} \subseteq \mathbb{R}$ be a parameterization of the regular orbit $\phi(t)$ between the base points of $\Sigma_{1}$ and $\Sigma_{2}$. Given a point $(x, y)$ in a sufficiently small neighborhood of the orbit $(\varphi(s), \psi(s))$, we can always encounter values of the curvilinear coordinates $(s, n)$ that realize the following change of variables: $x(s, n)=$ $\varphi(s)-n \psi^{\prime}(s), y(s, n)=\psi(s)+n \varphi^{\prime}(s)$. We remark that the variable $n$ measures the distance perpendicular to $\phi(t)$ from the point $(x, y)$ and, therefore, $n=0$ corresponds to the considered regular orbit $\phi(t)$. We can assume, without loss of generality, that the transversal section $\Sigma_{1}$ corresponds to $\Sigma_{1}:=\{s=0\}$ and $\Sigma_{2}$ to $\Sigma_{2}:=\{s=L\}$, for a certain real number $L>0$.

We perform the change to curvilinear coordinates $(x, y) \mapsto(s, n)$ in a neighborhood of the regular orbit $n=0$ with $s \in \mathcal{I}=[0, L]$. Then, system (1) reads

$$
\dot{n}=N(s, n), \quad \dot{s}=S(s, n),
$$

where $N(s, 0) \equiv 0$ since $n=0$ is an orbit and $S(s, 0) \neq 0$ for $s \in \mathcal{I}$ because it is a regular orbit. Therefore, and in order to study the behavior of the orbits in a neighborhood of $n=0$, we can consider the following ordinary differential equation:

$$
\frac{d n}{d s}=F(s, n) .
$$

We denote by $\Psi\left(s ; n_{0}\right)$ the flow associated to the equation (5) with initial condition $\Psi\left(0 ; n_{0}\right)=n_{0}$. In these coordinates, the Poincaré map $\Pi: \Sigma_{1} \rightarrow \Sigma_{2}$ between these two transversal sections is given by $\Pi\left(n_{0}\right)=\Psi\left(L ; n_{0}\right)$.

We assume the existence of an analytic inverse integrating factor $V(x, y)$ in a neighborhood of the considered regular orbit $\phi(t)$ of system (11). In fact, when $\Sigma_{1} \neq \Sigma_{2}$ and no return is involved, there always exists such an inverse integrating factor. It is clear that in a neighborhood of a regular point there is always an inverse integrating factor. Applying the method of characteristics, we can extend this function following the flow of the system until we find a singular point or a return is involved. 
The change to curvilinear coordinates gives us an inverse integrating factor for equation (5), denoted by $\tilde{V}(s, n)$, and which satisfies

$$
\frac{\partial \tilde{V}}{\partial s}+\frac{\partial \tilde{V}}{\partial n} F(s, n)=\frac{\partial F}{\partial n} \tilde{V}(s, n) .
$$

Remark 2. The relationship between $V(x, y)$ and $\tilde{V}(s, n)$ is

$$
\tilde{V}(s, n)=\frac{V(x(s, n), y(s, n))}{J(s, n) S(s, n)},
$$

where $J(s, n)$ is the Jacobian of the change $(x, y) \mapsto(s, n)$ to curvilinear coordinates and $S(s, n)$ comes from the time rescaling $t \mapsto s$ and is the function defined by system (41). We note that $J(s, n)=\varphi^{\prime}(s)^{2}+\psi^{\prime}(s)^{2}+O(n)$ and thus $J(s, 0) \neq 0$ for any $s \in \mathcal{I}$. Moreover, it is clear that $S(s, 0) \neq 0$ for all $s \in \mathcal{I}$.

The following theorem gives the relation between the inverse integrating factor and the Poincaré map defined over the considered regular orbit.

Theorem 3. We consider a regular orbit $\phi(t)$ of system (11) which has an inverse integrating factor $V(x, y)$ of class $\mathcal{C}^{1}$ defined in a neighborhood of it and we consider the Poincaré map associated to the regular orbit between two transversal sections $\Pi: \Sigma_{1} \rightarrow \Sigma_{2}$. We perform the change to curvilinear coordinates and we consider the ordinary differential equation (5) with the inverse integrating factor $\tilde{V}(s, n)$ which is obtained from $V(x, y)$. In these coordinates, the transversal sections can be taken such that $\Sigma_{1}:=\{s=0\}$ and $\Sigma_{2}:=\{s=L\}$, for a certain real value $L>0$. We parameterize $\Sigma_{1}$ by the real value of the coordinate $n$. The following identity holds:

$$
\tilde{V}(L, \Pi(n))=\tilde{V}(0, n) \Pi^{\prime}(n) .
$$

The proof of this result is given in Section 2, We remark that if we know an inverse integrating factor for system (11), we can construct the function $\tilde{V}(s, n)$ and equation (7) gives an ordinary differential equation for $\Pi(n)$, which is always of separable variables. Thus, we can determine the expression of $\Pi(n)$ in an implicit way and up to quadratures, whereas the definition of the Poincaré map $\Pi$ is geometric. As far as the authors know, no such way of describing the Poincaré map has been given in any previous work. The first example in Section 3 illustrates this remark.

It is well known, due to the flow box theorem, that a regular orbit of system (11) which is not a separatrix of singular points nor a limit cycle has an associated Poincaré map conjugated with the identity. There are several results which establish that the vanishing set of an inverse integrating factor gives the orbits whose associated Poincaré map is not conjugated with the identity. In this sense, Giacomini, Llibre and Viano [13] showed that any limit cycle $\gamma \subset \mathcal{U} \subseteq \mathbb{R}^{2}$ of system (11) satisfies $\gamma \subset V^{-1}(0)$ provided that the inverse integrating factor $V$ is defined in $\mathcal{U}$.

Lately it was shown that the zero-set of $V$ often contains the separatrices of critical points in $\mathcal{U}$. More precisely, in [3], Berrone and Giacomini proved that, if $p_{0}$ is a hyperbolic saddle point of system (11), then any inverse integrating factor $V$ defined in a neighborhood of $p_{0}$ vanishes on all four separatrices of $p_{0}$, provided $V\left(p_{0}\right)=0$. We emphasize here that this result does not hold, in general, for nonhyperbolic singularities (see [1] for example).

We are going to consider regular orbits whose Poincaré map is a return map. We take profit from the result stated in Theorem 3 in order to study the Poincaré map 
associated to a limit cycle or to a homoclinic loop, in terms of the inverse integrating factor. Although we have used curvilinear coordinates to state Theorem 3, we do not need to use them to establish the results described in the following Theorems 4 and 6. The only hypothesis that we need is the existence and the expression of an analytic inverse integrating factor in a neighborhood of the limit cycle or the homoclinic loop. In particular, we are able to give the cyclicity of a limit cycle or of a homoclinic loop from the vanishing multiplicity of the inverse integrating factor over the limit cycle or the homoclinic loop. The vanishing multiplicity of an analytic inverse integrating factor $V(x, y)$ of system (11) over a regular orbit $\phi(t)$ is defined as follows. We recall the local change of coordinates $x(s, n)=\varphi(s)-n \psi^{\prime}(s)$, $y(s, n)=\psi(s)+n \varphi^{\prime}(s)$ defined in a neighborhood of the considered regular orbit $n=0$. If we have the following Taylor development around $n=0$ :

$$
V(x(s, n), y(s, n))=n^{m} v(s)+O\left(n^{m+1}\right),
$$

where $m$ is an integer with $m \geq 0$ and the function $v(s)$ is not identically null, we say that $V$ has multiplicity $m$ on $\phi(t)$. In fact, as we will see in Lemma 7, $v(s) \neq 0$ for any $s \in \mathcal{I}$, and thus, the vanishing multiplicity of $V$ on $\phi(t)$ is well defined over all its points.

Let us consider as regular orbit a limit cycle $\gamma$ and use the parameterization of $\gamma$ in curvilinear coordinates $(s, n)$ with $s \in[0, L)$. The Poincaré return map $\Pi$ associated to $\gamma$ coincides with the previously defined map in which $\Sigma:=\Sigma_{1}=$ $\Sigma_{2}$. Since the qualitative properties of $\Pi$ do not depend on the chosen transversal section $\Sigma$, we take the one given by the curvilinear coordinates and, thus, $\Pi\left(n_{0}\right)=$ $\Psi\left(L ; n_{0}\right)$. It is well known that $\Pi$ is analytic in a neighborhood of $n_{0}=0$. We recall that the periodic orbit $\gamma$ is a limit cycle if, and only if, the Poincaré return map $\Pi$ is not the identity. If $\Pi$ is the identity, we have that $\gamma$ belongs to a period annulus. We recall the definition of multiplicity of a limit cycle: $\gamma$ is said to be a limit cycle of multiplicity 1 if $\Pi^{\prime}(0) \neq 1$ and $\gamma$ is said to be a limit cycle of multiplicity $m$ with $m \geq 2$ if $\Pi\left(n_{0}\right)=n_{0}+\beta_{m} n_{0}^{m}+O\left(n_{0}^{m+1}\right)$ with $\beta_{m} \neq 0$. The following result states that given a limit cycle $\gamma$ of (1) with multiplicity $m$, then the vanishing multiplicity of an analytic inverse integrating factor defined in a neighborhood of it must also be $m$.

Theorem 4. Let $\gamma$ be a periodic orbit of system (1) and let $V$ be an analytic inverse integrating factor defined in a neighborhood of $\gamma$.

(a) If $\gamma$ is a limit cycle of multiplicity $m$, then $V$ has vanishing multiplicity $m$ on $\gamma$.

(b) If $V$ has vanishing multiplicity $m$ on $\gamma$, then $\gamma$ is a limit cycle of multiplicity $m$ or it belongs to a continuum of periodic orbits.

The proof of this theorem is given in Section 2 ,

The idea of studying the multiplicity of a limit cycle by means of the vanishing multiplicity of the inverse integrating factor already appears in the work [14. In that work the considered limit cycles are semistable and the vanishing multiplicity is defined in terms of polar coordinates, which only apply for convex limit cycles.

Since the Poincaré map of a periodic orbit is an analytic function and the multiplicity of a limit cycle is a natural number, we obtain the following corollary from the previous result. 
Corollary 5. Let $\gamma$ be a periodic orbit of system (11) and let $V$ be an inverse integrating factor of class $\mathcal{C}^{1}$ defined in a neighborhood of $\gamma$. We take the change to curvilinear coordinates $x(s, n)=\varphi(s)-n \psi^{\prime}(s), y(s, n)=\psi(s)+n \varphi^{\prime}(s)$ defined in a neighborhood of $\gamma$. If we have that the leading term in the following development around $n=0$ :

$$
V(x(s, n), y(s, n))=n^{\rho} v(s)+o\left(n^{\rho}\right),
$$

where $v(s) \not \equiv 0$, is such that either $\rho=0$ or $\rho>1$ and $\rho$ is not a natural number, then $\gamma$ belongs to a continuum of periodic orbits.

We remark that this corollary applies, for instance, in the following case: let us consider an invariant curve $f=0$ of system (1) with an oval $\gamma$ such that $\left.\nabla f\right|_{\gamma} \neq 0$ and let us assume that $\gamma$ corresponds to a periodic orbit of the system and that there exists an inverse integrating factor of the form $V=f^{\rho} g$, where $g$ is a nonzero function of class $\mathcal{C}^{1}$ in a neighborhood of $\gamma$ and $\rho \in \mathbb{R}$, with either $\rho=0$ or $\rho \geq 1$ in order to have a $\mathcal{C}^{1}$ inverse integrating factor in a neighborhood of $\gamma$. If we have that either $\rho=0$ or $\rho$ is not a natural number, then we deduce that $\gamma$ belongs to a period annulus, by Corollary 5 . On the other hand, applying Theorem 4 , in the case that $\rho=m \in \mathbb{N}$, we deduce that either $\gamma$ belongs to a period annulus or it is a limit cycle with multiplicity $m$.

A regular orbit $\phi(t)=(x(t), y(t))$ of (11) is called a homoclinic orbit if $\phi(t) \rightarrow p_{0}$ as $t \rightarrow \pm \infty$ for some singular point $p_{0}$. We emphasize that such kinds of orbits arise in the study of bifurcation phenomena as well as in many applications in several sciences. A homoclinic loop is the union $\Gamma=\phi(t) \cup\left\{p_{0}\right\}$. We assume that $p_{0}$ is a hyperbolic saddle, that is, a critical point of system (1) such that the eigenvalues of the Jacobian matrix $D \mathcal{X}\left(p_{0}\right)$ are both real, different from zero and of opposite signs. We remark that this type of graphic always has associated (maybe only its inner or outer neighborhood) a Poincaré return map $\Pi: \Sigma \rightarrow \Sigma$ with $\Sigma$ any local transversal section through a regular point of $\Gamma$. Moreover, in this work we only study compact homoclinic loops that are the $\alpha$ - or $\omega$-limit set of the points in its neighborhood, i.e., homoclinic loops with a finite singular point and a return map different from the identity. This fact implies that $\Gamma$ is a compact set, that is, it does not have intersection with the equator in the Poincaré compactification. In fact the same results can be used for some homoclinic loops whose saddle is in the equator of the Poincaré compactification, but we always assume that the affine chart we are working with completely contains the homoclinic loop, that is, the homoclinic loops we take into account are compact in the considered affine chart.

Our goal in this work is to study the cyclicity of the described homoclinic loop $\Gamma$ in terms of the vanishing multiplicity of an inverse integrating factor. Roughly speaking, the cyclicity of $\Gamma$ is the maximum number of limit cycles which bifurcate from it under a smooth perturbation of (11); see 21] for a precise definition. The study of the cyclicity of $\Gamma$ has been tackled by the comparison between the Poincaré return map of the unperturbed system (1) and that of the perturbed system in terms of the perturbation parameters. The main result in this study is due to Roussarie and appears in [21]. Roussarie presents the asymptotic expression of the Poincaré map associated to the homoclinic loop $\Gamma$, which allows us to characterize the cyclicity of $\Gamma$. We recall this result in Section 2, A good book on the subject where these and other results are stated with proofs is [22. The way we contribute to the study of the cyclicity of $\Gamma$ is based on the use of inverse integrating factors. 
We take profit from the result of Roussarie to characterize the cyclicity of $\Gamma$ as we are able to relate the Poincaré return map with the inverse integrating factor.

The Poincaré map $\Pi: \Sigma \rightarrow \Sigma$ associated to a homoclinic loop $\Gamma=\phi(t) \cup\left\{p_{0}\right\}$ is given as the composition of the transition map along the regular homoclinic orbit $\phi(t)$, which we denote by $R$, with the transition map in a neighborhood of the saddle point $p_{0}$, which we denote by $\Delta$. We recall that $\Sigma$ is a transversal section based on a regular point of $\Gamma$. We have that $R$ is an analytic diffeomorphism defined in a neighborhood of the regular part $\phi(t)$ of $\Gamma$. Hence, in this context, $R$ coincides with the Poincaré map associated along the whole $\phi(t)$ and, therefore, it satisfies the identity described in Theorem 3. The map $\Delta$ is the transition map defined in a neighborhood of the critical point $p_{0}$. This map $\Delta$ is characterized by the first nonvanishing saddle quantity associated to $p_{0}$. We recall that the first saddle quantity is $\alpha_{1}=\operatorname{div} \mathcal{X}\left(p_{0}\right)$ and it classifies the point $p_{0}$ between being strong (when $\alpha_{1} \neq 0$ ) or weak (when $\alpha_{1}=0$ ). If $p_{0}$ is a weak saddle point, the saddle quantities are the obstructions for it to be analytically orbitally linearizable. We give the definition of a saddle point to be analytically orbitally linearizable in Section 2 .

In order to define the saddle quantities associated to $p_{0}$, we translate the saddle point $p_{0}$ to the origin of coordinates and we make a linear change of variables so that its unstable (resp. stable) separatrix has the horizontal (resp. vertical) direction at the origin. Let $p_{0}$ be a weak hyperbolic saddle point situated at the origin of coordinates and whose associated eigenvalues are taken to be \pm 1 by a rescaling of time, if necessary. Then, see for instance [19], the existence is well known of an analytic near-identity change of coordinates that brings the system into:

$$
\begin{aligned}
& \dot{x}=x+\sum_{i=1}^{k-1} a_{i} x^{i+1} y^{i}+a_{k} x^{k+1} y^{k}+\cdots, \\
& \dot{y}=-y-\sum_{i=1}^{k-1} a_{i} x^{i} y^{i+1}-b_{k} x^{k} y^{k+1}+\cdots,
\end{aligned}
$$

with $a_{k}-b_{k} \neq 0$ and where the dots denote terms of higher order. The first nonvanishing saddle quantity is defined by $\alpha_{k+1}:=a_{k}-b_{k}$. We remark that the first nonvanishing saddle quantity can be obtained through an algebraic algorithm.

We consider a homoclinic loop $\Gamma=\phi(t) \cup\left\{p_{0}\right\}$ where $\phi(t)$ is a homoclinic orbit through the hyperbolic saddle point $p_{0}$. We assume that there exists an analytic inverse integrating factor $V$ defined in a neighborhood of the homoclinic loop $\Gamma$. The following theorem establishes the relation between the vanishing multiplicity of the inverse integrating factor $V$ over the homoclinic orbit $\phi(t)$ and the cyclicity of $\Gamma$. We remark that the vanishing multiplicity of the inverse integrating factor also allows us to determine the first nonvanishing saddle quantity associated to the hyperbolic saddle point $p_{0}$, in case it exists.

Theorem 6. Let $\Gamma$ be a compact homoclinic loop through the hyperbolic saddle point $p_{0}$ of system (11) whose Poincaré return map is not the identity. Let $V$ be an analytic inverse integrating factor defined in a neighborhood of $\Gamma$ with vanishing multiplicity $m$ over $\Gamma$. Then, $m \geq 1$ and the first possible nonvanishing saddle quantity is $\alpha_{m}$. Moreover,

(i) the cyclicity of $\Gamma$ is $2 m-1$, if $\alpha_{m} \neq 0$,

(ii) the cyclicity of $\Gamma$ is $2 m$, otherwise. 
This theorem is proved in Section 2 Theorems 3 and 6 are the main results presented in this work. We consider the following situation: we have an analytic system (11) with a compact homoclinic loop $\Gamma$ through the hyperbolic saddle point $p_{0}$ and whose Poincaré return map is not the identity and we aim at determining the cyclicity of $\Gamma$. Theorem 6 gives us an algorithmic procedure to solve this problem, provided we know an analytic inverse integrating factor $V$ of (1) in a neighborhood of $\Gamma$ with vanishing multiplicity $m$ over it. We compute the $m^{\text {th }}$ saddle quantity $\alpha_{m}$ at $p_{0}$ recalling that all the previous saddle quantities vanish. If $\alpha_{m} \neq 0$, then the cyclicity of $\Gamma$ is $2 m-1$. Otherwise, when $\alpha_{m}=0$, the cyclicity of $\Gamma$ is $2 m$.

We notice that the determination of the $m^{\text {th }}$ saddle quantity can be overcome through an algebraic procedure. Thus, our result also applies in the particular case in which the saddle of the homoclinic loop is linearizable, that is, the case in which a bound for the cyclicity of this graphic cannot be determined through an algebraic method.

We remark that the same result can be applied to a double homoclinic graphic, that is, two homoclinic orbits which share the same hyperbolic saddle point $p_{0}$. This kind of graphic has also been treated in [16, 17. To study the perturbation of Hamiltonian systems with a homoclinic loop or a double homoclinic loop, which is a topic not addressed by the present paper, see [18 and the references therein.

This paper is organized as follows. The next section contains the proof of the main results, which are stated in this first section. Moreover, in Section 2, several additional results appear, related with the problems of studying the cyclicity of a limit cycle or a homoclinic loop. These results allow the proof of the main results and are interesting by themselves as they give the state-of-the-art of the aforementioned problems. Much of the results that we present are generalizations of previous ones and can be better understood within the context of this work. The last section contains several examples which illustrate and complement our results.

\section{AdDitional RESUlts AND PROOFS}

We consider a regular orbit of system (1) and we are going to prove the result we have about it, Theorem 3. We consider the aforementioned transition map $\Pi: \Sigma_{1} \rightarrow \Sigma_{2}$ along the regular orbit. The key tool for studying this Poincaré map is to change the coordinates of system (11) to local coordinates in a neighborhood of the regular orbit, that is, to change to the defined curvilinear coordinates $(s, n)$. We recall that $n=0$ denotes the considered regular orbit and $s \in \mathcal{I} \subset \mathbb{R}$ gives the transition along the orbits since the two transversal sections can be taken as $\Sigma_{1}=\{s:=0\}$ and $\Sigma_{2}=\{s:=L\}$, with $L$ a strictly positive real value. These coordinates also allow us to give a definition for the Poincaré map in terms of the flow $\Psi\left(s ; n_{0}\right)$ associated with the ordinary differential equation (5) as $\Pi\left(n_{0}\right)=$ $\Psi\left(L ; n_{0}\right)$. We remark that the expression of the flow $\Psi\left(s ; n_{0}\right)$ in a neighborhood of the considered orbit $n_{0}=0$ can be encountered by means of recursive formulae at each order of $n_{0}$. This recursive determination of the flow $\Psi\left(s ; n_{0}\right)$ allows the study of the Poincaré map $\Pi\left(n_{0}\right)$. However, these recursive formulae involve iterated integrals which can be, and usually are, very difficult to compute. The explanation of this process and an application to the study of the multiplicity of a limit cycle can be encountered in the work [12].

We assume the existence of an inverse integrating factor $V(x, y)$ of class $\mathcal{C}^{1}$ defined in a neighborhood of the regular orbit of system (1). Then, we can construct 
an inverse integrating factor for the equation (5), which we denote by $\tilde{V}(s, n)$ and which satisfies the partial differential equation (6). Moreover, the change to curvilinear coordinates gives the definition of vanishing multiplicity of $V(x, y)$ over the considered regular orbit, as described through the expression (8).

The following auxiliary lemma gives us that the vanishing multiplicity of $V$ over the regular orbit is well defined on all its points.

Lemma 7. The function $v(s)$ appearing in (8) is different from zero for any $s \in \mathcal{I}$.

Proof. First, by Remark 2, we have $\tilde{V}(s, n)=V(x(s, n), y(s, n)) /(J(s, n) S(s, n))$. The Taylor development of $\tilde{V}(s, n)$ around $n=0$ is of the form

$$
\tilde{V}(s, n)=\tilde{v}(s) n^{m}+O\left(n^{m+1}\right) .
$$

Here, $\tilde{v}(s)=j(s) v(s)$, where $j(s)=1 /(J(s, 0) S(s, 0)) \neq 0$ for any $s \in \mathcal{I}$.

To end with, we are going to see that $\tilde{v}(s) \neq 0$ for any $s \in \mathcal{I}$. We recall that, by assumption, $0 \in \mathcal{I}$. Since $\tilde{V}(s, n)$ satisfies equation (6), we deduce that:

$$
\tilde{V}\left(s, \Psi\left(s ; n_{0}\right)\right)=\tilde{V}\left(0, \Psi\left(0 ; n_{0}\right)\right) \exp \left\{\int_{0}^{s} \frac{\partial F}{\partial n}\left(\sigma, \Psi\left(\sigma ; n_{0}\right)\right) d \sigma\right\} .
$$

We develop this identity around $n_{0}=0$ and we get that

$$
\tilde{v}(s) n_{0}^{m}+O\left(n_{0}^{m+1}\right)=\tilde{v}(0) \exp \left\{\int_{0}^{s} \frac{\partial F}{\partial n}(\sigma, 0) d \sigma\right\} n_{0}^{m}+O\left(n_{0}^{m+1}\right) .
$$

Since $\tilde{v}(s) \not \equiv 0$, we can assume $\tilde{v}(0) \neq 0$. Therefore, equating the coefficients of $n_{0}^{m}$ in both members of the previous identity, we have the desired result.

We have stated all the definitions and notation needed to give the proof of Theorem 3 ,

Proof of Theorem 3. We have that $\Psi\left(s ; n_{0}\right)$ satisfies

$$
\frac{\partial \Psi}{\partial s}\left(s ; n_{0}\right)=F\left(s, \Psi\left(s ; n_{0}\right)\right) \text { and } \Psi\left(0 ; n_{0}\right)=n_{0},
$$

as it is the flow of equation (5) with initial condition $n_{0}$. We differentiate the previous identities with respect to $n_{0}$ and we have that:

$$
\frac{\partial}{\partial s}\left(\frac{\partial \Psi}{\partial n_{0}}\left(s ; n_{0}\right)\right)=\frac{\partial F}{\partial n}\left(s, \Psi\left(s ; n_{0}\right)\right)\left(\frac{\partial \Psi}{\partial n_{0}}\left(s ; n_{0}\right)\right) \text { and } \frac{\partial \Psi}{\partial n_{0}}\left(0 ; n_{0}\right)=1 .
$$

Hence,

$$
\int_{0}^{s} \frac{\partial F}{\partial n}\left(\sigma, \Psi\left(\sigma ; n_{0}\right)\right) d \sigma=\int_{0}^{s} \frac{\frac{\partial}{\partial \sigma}\left(\frac{\partial \Psi}{\partial n_{0}}\left(\sigma ; n_{0}\right)\right)}{\frac{\partial \Psi}{\partial n_{0}}\left(\sigma ; n_{0}\right)} d \sigma=\left.\ln \left(\frac{\partial \Psi}{\partial n_{0}}\left(\sigma ; n_{0}\right)\right)\right|_{\sigma=0} ^{\sigma=s} .
$$

Since $\frac{\partial \Psi}{\partial n_{0}}\left(0 ; n_{0}\right)=1$, we deduce that

$$
\int_{0}^{s} \frac{\partial F}{\partial n}\left(\sigma, \Psi\left(\sigma ; n_{0}\right)\right) d \sigma=\ln \left(\frac{\partial \Psi}{\partial n_{0}}\left(s ; n_{0}\right)\right) .
$$

Using that the function $\tilde{V}(s, n)$ satisfies (10), we conclude that

$$
\tilde{V}\left(s, \Psi\left(s ; n_{0}\right)\right)=\tilde{V}\left(0, \Psi\left(0 ; n_{0}\right)\right) \frac{\partial \Psi}{\partial n_{0}}\left(s ; n_{0}\right) .
$$

When we take $s=L$ and we recall that the Poincaré map is defined as $\Pi\left(n_{0}\right)=$ $\Psi\left(L ; n_{0}\right)$, we deduce identity (7). 
Let us consider as a regular orbit a limit cycle $\gamma$ and use the parameterization of the whole $\gamma$ in curvilinear coordinates with $s \in[0, L)$. The value of $L$ can be taken to be the length of the limit cycle $\gamma$, and $s$ can be the arc-length parameter associated to it. The Poincaré return map $\Pi$ associated to $\gamma$ coincides with the previously defined map in which $\Sigma:=\Sigma_{1}=\Sigma_{2}$. We remark that in curvilinear coordinates $\Sigma_{1}$ corresponds to the value $s=0$ and $\Sigma_{2}$ to the value $s=L$ and that these two sections are equal only in coordinates $(x, y)$. Since $\gamma$ is a periodic orbit, the change to curvilinear coordinates means that all the analytic functions in $(x, y)$ in a neighborhood of $\gamma$ need to be $L$-periodic in $s$.

We use that $\tilde{V}(s, n)$ is $L$-periodic in $s$ and we develop relation (7) in a neighborhood of $n=0$ in order to get the following result. We recall that the point $n=0$ corresponds to the periodic orbit $\gamma$ and that it is, therefore, a fixed point of its Poincaré map.

Proposition 8. Let us consider the following Taylor development of the analytic Poincaré map $\Pi(n)$ associated to a periodic orbit $\gamma$, around $n=0$ :

$$
\Pi(n)=\left\{\begin{array}{lll}
\mathrm{e}^{\beta_{1}} n+O\left(n^{2}\right) & \text { with } & \beta_{1} \neq 0, \text { or } \\
n+\beta_{k} n^{k}+O\left(n^{k+1}\right) & \text { with } & \beta_{k} \neq 0, k>1, \text { or } \\
n & \text { otherwise. } &
\end{array}\right.
$$

Let $m \geq 0$ be the vanishing multiplicity of an analytic inverse integrating factor $V$ on $\gamma$.

(a) If $m=0$, then $\beta_{k}=0$ for all $k \geq 1$.

(b) If $m \geq 1$, then either $m$ is the lowest subindex such that $\beta_{m} \neq 0$ or $\beta_{k}=0$ for all $k \geq 1$.

Proof. Let us recall the Taylor development of the function $\tilde{V}\left(s, n_{0}\right)$ in a neighborhood of $n_{0}=0$ : $\tilde{V}\left(s, n_{0}\right)=\sum_{i \geq 0} \tilde{v}_{i}(s) n_{0}^{i}$. Since the function $V(x, y)$ is not locally null, we have that there exists an integer $m$ with $m \geq 0$ which is the lowest index with $\tilde{v}_{m}(s) \neq 0$ for any $s \in \mathcal{I}$ from Lemma 7. By definition, this value of $m$ is the vanishing multiplicity of $V(x, y)$ on the considered regular orbit.

Since $V(x, y)$ changed to local coordinates is $L$-periodic in $s$, we have that the function $\tilde{V}(s, n)$ is also $L$-periodic in $s$. Then, we can write $\tilde{V}(0, n)=\sum_{i \geq 0} \nu_{i} n^{i}$ and $\tilde{V}(L, \Pi(n))=\sum_{i \geq 0} \nu_{i} \Pi(n)^{i}$.

If $\beta_{1} \neq 0$, we evaluate identity (7) in $n=0$ and we get that $\tilde{V}(L, 0)=$ $\mathrm{e}^{\beta_{1}} \tilde{V}(0,0)$, which implies that $\nu_{0}=0$ and, thus, the vanishing multiplicity of $V(x, y)$ on the regular orbit is at least 1 . We develop identity (7) in powers of $n$ and the lowest order terms in both members of the equality correspond to $n^{m}$. The equation for their coefficients is: $\nu_{m} \mathrm{e}^{m \beta_{1}}=\nu_{m} \mathrm{e}^{\beta_{1}}$. Since $\beta_{1} \neq 0$, we deduce that $m=1$.

Let $k>1$ be the lowest subindex such that $\beta_{k} \neq 0$. We have that $\Pi(n)=$ $n+\beta_{k} n^{k}+O\left(n^{k+1}\right)$. We subtract $\tilde{V}(0, n)$ from both members of (17) and we get the following relation:

$$
\sum_{i \geq 1} \nu_{i}\left(\Pi(n)^{i}-n^{i}\right)=\left(\nu_{0}+\sum_{i \geq 1} \nu_{i} n^{i}\right)\left(k \beta_{k} n^{k-1}+O\left(n^{k}\right)\right) .
$$

The left hand side of (11) has order at least $n^{k}$, which implies that $\nu_{0}=0$, in order to have the same order in both members. Therefore, $m>0$. We have that the 
lowest order terms in both sides of (11) correspond to $n^{m+k-1}$ and the equation of their coefficients is: $m \beta_{k} \nu_{m}=k \beta_{k} \nu_{m}$, which implies that $k=m$.

Proof of Theorem 4. The proof is a straightforward consequence of the previous Proposition 8. We have preferred to state this proposition as we are going to use the same reasoning for the transition map associated to a homoclinic orbit.

Let us now consider a homoclinic loop $\Gamma=\phi(t) \cup\left\{p_{0}\right\}$ whose critical point $p_{0}$ is a hyperbolic saddle point. We will denote by $\lambda$ and $\mu$ the eigenvalues associated to the Jacobian matrix $D \mathcal{X}\left(p_{0}\right)$ with the convention $\mu<0<\lambda$. We associate to $p_{0}$ its hyperbolicity ratio $r=-\mu / \lambda$. We say that the singular point $p_{0}$ is strong if $\operatorname{div} \mathcal{X}\left(p_{0}\right) \neq 0$ (equivalently $r \neq 1$ ) and it is weak otherwise. The hyperbolic saddle $p_{0}$ is called $p: q$ resonant if $r=q / p \in \mathbb{Q}^{+}$with $p$ and $q$ natural and coprime numbers.

A homoclinic loop $\Gamma$ is called stable (unstable) if all the trajectories in some inner or outer neighborhood of $\Gamma$ approach $\Gamma$ as $t \rightarrow+\infty(t \rightarrow-\infty)$. In the investigation of the stability of a homoclinic loop $\Gamma$ of system (11) through a saddle $p_{0}$ the quantity

$$
\alpha_{1}=\operatorname{div} \mathcal{X}\left(p_{0}\right)
$$

plays an important role. In short, it is well known, see for instance p. 304 of [1, that $\Gamma$ is stable (unstable) if $\alpha_{1}<0\left(\alpha_{1}>0\right)$. For this reason such kinds of homoclinic loops $\Gamma$ are said to be simple if $\alpha_{1} \neq 0$ and multiple otherwise.

The cyclicity of $\Gamma$ is linked with its stability. It is notable to observe that, in the simple case $\alpha_{1} \neq 0$, the stability of $\Gamma$ is only determined by the nature of the saddle point itself. Andronov et al. [1] proved that if $\alpha_{1} \neq 0$, the possible limit cycle that bifurcates from $\Gamma$ after perturbing the system by a multiparameter family has the same type of stability as $\Gamma$; hence this limit cycle is unique for small values of the parameters. After that, Cherkas [8] showed that, if $\alpha_{1}=0$ and the associated Poincaré map of the loop $\Gamma$ is hyperbolic, then the maximum number of limit cycles that can appear near $\Gamma$ perturbing the system in the $\mathcal{C}^{1}$ class is 2 . We recall that a real map is said to be hyperbolic at a point if its derivative at the point has modulus different from 1. In 21, Roussarie presents a generalization of these results which determine the asymptotic expression of the Poincaré map associated to the loop $\Gamma$.

The Poincaré map $\Pi$ associated to $\Gamma$ is defined over a transversal section $\Sigma$ whose base point is a regular point of $\Gamma$. We parameterize the transversal section $\Sigma$ by a real local coordinate $\sigma$. The value $\sigma=0$ corresponds to the intersection of $\Sigma$ with $\Gamma$ and $\sigma>0$ is the side of $\Gamma$ where $\Pi(\sigma)$ is defined. Roussarie's result is twofold: on the one hand, the asymptotic expansion of $\Pi(\sigma)$ is determined and, on the other hand, the cyclicity of $\Gamma$ is deduced from it.

Theorem 9 ([21]). Let us consider any smooth perturbation of system (11). Then we have:

(i) If $r \neq 1$ (equivalently, $\alpha_{1} \neq 0$ ), then $\Pi(\sigma)=c \sigma^{r}(1+o(1))$ with $c>0$ and at most 1 limit cycle can bifurcate from $\Gamma$.

(ii) If $\alpha_{1}=0$ and $\beta_{1} \neq 0$, then $\Pi(\sigma)=\mathrm{e}^{\beta_{1}} \sigma+o(\sigma)$ and at most 2 limit cycles can bifurcate from $\Gamma$.

(iii) If $\alpha_{i}=\beta_{i}=0$ for $i=1,2, \ldots, k$ with $k \geq 1$ and $\alpha_{k+1} \neq 0$, then $\Pi(\sigma)=$ $\sigma+\alpha_{k+1} \sigma^{k+1} \log \sigma+o\left(\sigma^{k+1} \log \sigma\right)$ and at most $2 k+1$ limit cycles can bifurcate from $\Gamma$. 
(iv) If $\alpha_{i}=\beta_{i}=\alpha_{k}=0$ for $i=1,2, \ldots, k-1$ with $k>1$ and $\beta_{k} \neq 0$, then $\Pi(\sigma)=\sigma+\beta_{k} \sigma^{k}+o\left(\sigma^{k}\right)$ and at most $2 k$ limit cycles can bifurcate from $\Gamma$.

(v) If $\alpha_{i}=\beta_{i}=0$ for all $i \geq 1$, then $\Pi(\sigma)=\sigma$ and the number of limit cycles that can bifurcate from $\Gamma$ has no upper bound.

We remark that identity $\Pi(\sigma)=\sigma$ in statement (v) of Theorem 9 is just formally true. Moreover, in this case, the fact that we do not fix the possible perturbation of system (11) inside a certain family gives that there is no upper bound on the cyclicity of $\Gamma$.

The values $\alpha_{i}$, with $i \geq 1$, are the saddle quantities associated to $p_{0}$ and are evaluated by a local computation. The values $\beta_{i}$, with $i \geq 1$, are called separatrix quantities of $\Gamma$ and correspond to a global computation. The determination of the $\alpha_{i}$ can be explicitly done through the algebraic method of normal form theory near $p_{0}$. Additionally, $\beta_{1}=\int_{\Gamma} \operatorname{div} \mathcal{X} d t$, and a more complicated expression for $\beta_{2}$ can be encountered in [16] and it involves several iterated integrals. On the contrary, as far as we know, there is no closed form expression to get $\beta_{i}$ for $i \geq 3$. We remark that our result provides a way to determine the cyclicity of the homoclinic loop $\Gamma$ without computing any $\beta_{i}$.

A graphic $\bar{\Gamma}=\bigcup_{i=1}^{k} \phi_{i}(t) \cup\left\{p_{1}, \ldots, p_{k}\right\}$ is formed by $k$ singular points $p_{1}, \ldots, p_{k}$, $p_{k+1}=p_{1}$ and $k$ oriented regular orbits $\phi_{1}(t), \ldots, \phi_{k}(t)$, connecting them such that $\phi_{i}(t)$ is an unstable characteristic orbit of $p_{i}$ and a stable characteristic orbit of $p_{i+1}$. A graphic may or may not have associated a Poincaré return map. In case it has one, it is called a polycycle. Of course, the homoclinic loops that we consider are polycycles with just one singular point. In [3], the case of a system (1) with a compact polycycle $\bar{\Gamma}$ whose associated Poincaré map is not the identity and whose critical points $p_{1}, p_{2}, \ldots, p_{k}$ are all nondegenerate is studied and it is shown that if there exists an inverse integrating factor $V$ defined in a neighborhood of $\bar{\Gamma}$, then $\bar{\Gamma} \subseteq V^{-1}(0)$. A strong generalization of this result is given in [11, where it is shown that any compact polycycle contained in $\mathcal{U}$ with nonidentity Poincaré return map is contained into the zero-set of $V$ under mild conditions. More concretely, García and Shafer give the following result.

Theorem 10 ([1]). Assume the existence of an analytic inverse integrating factor $V$ defined in a neighborhood $\mathcal{N}$ of any compact polycycle $\bar{\Gamma}$ of system (1) with associated Poincaré return map different from the identity. Then, $\bar{\Gamma} \subset V^{-1}(0)$.

In [11, this result is also given for an inverse integrating factor $V$ with lower regularity than analytic and assuming several conditions. In fact, a consequence of their results is that if we consider a compact homoclinic loop $\Gamma$ whose Poincaré map is not the identity and such that there exists an inverse integrating factor $V$ of class $\mathcal{C}^{1}$ defined in a neighborhood of $\Gamma$, then $\Gamma \subset V^{-1}(0)$.

In this paper, we will work with inverse integrating factors $V(x, y)$ analytic in a neighborhood of a compact homoclinic loop $\Gamma=\phi(t) \cup\left\{p_{0}\right\}$ with associated Poincaré map different from the identity. Hence, $\Gamma \subset V^{-1}(0)$.

Regarding the existence problem, it is well known that the partial differential equation (2) has a solution in a neighborhood of any regular point, but not necessarily elsewhere. In [6] it is shown that if system (11) is analytic in a neighborhood $\mathcal{N} \subset \mathcal{U}$ of a critical point that is either a strong focus, a nonresonant hyperbolic node, or a Siegel hyperbolic saddle, then there exists a unique analytic inverse integrating factor on $\mathcal{N}$ up to a multiplicative constant. Hence, we only have at the 
moment the local existence of inverse integrating factors in a neighborhood of convenient singular points. But, we shall need to know a priori whether there exists an inverse integrating factor $V$ defined on a neighborhood of the whole homoclinic loop $\Gamma$ of system (11). This is a hard nonlocal problem of existence of global solutions of the partial differential equation (2) for which we do not know its answer. We describe one obstruction to the existence of an analytic inverse integrating factor defined in a neighborhood of certain homoclinic loops.

Proposition 11. Suppose that system (1) has a homoclinic loop $\Gamma$ through the hyperbolic saddle point $p_{0}$ which is not orbitally linearizable, $p: q$ resonant and strong $(p \neq q)$. Then, there is no analytic inverse integrating factor $V(x, y)$ defined in a neighborhood of $\Gamma$.

Proof. Let us assume that there exists an analytic inverse integrating factor $V(x, y)$ defined in a neighborhood of $\Gamma$. Let us consider $f_{\lambda}(x, y)=0$ and $f_{\mu}(x, y)=0$, the local analytic expressions of each of the separatrices associated to $p_{0}$, where the subindex denotes the corresponding eigenvalue. As we will see in Proposition 13 (iii), we have that $V(x, y)$ factorizes, as an analytic function in a neighborhood of $p_{0}$, in the form $V=f_{\lambda}^{1+k p} f_{\mu}^{1+k q} u$ with $u\left(p_{0}\right) \neq 0$ and an integer $k \geq 0$. Since $V(x, y)$ is defined on the whole loop $\Gamma$, it needs to have the same vanishing multiplicity on each separatrix at $p_{0}$, which implies that $k=0$. Thus, we have that the local factorization of $V$ in a neighborhood of $p_{0}$ is $V=f_{\lambda} f_{\mu} u$.

Let us take local coordinates in a neighborhood of $p_{0}$, which we assume to be at the origin. We recall, see 2] and the references therein, that given an analytic system $\dot{x}=\lambda x+\cdots, \dot{y}=\mu y+\cdots$ near the origin with $\mu / \lambda=-q / p \in \mathbb{Q}^{-}$with $p$ and $q$ natural and coprime numbers ( $p: q$ resonant saddle), then the system has two analytic invariant curves passing through the origin. Moreover, it is formally orbitally equivalent to

$$
\dot{X}=p X\left[1+\delta\left(U^{\ell}+a U^{2 \ell}\right)\right], \quad \dot{Y}=-q Y,
$$

with $U=X^{q} Y^{p}, a \in \mathbb{R}, \ell$ an integer such that $\ell \geq 1$ and $\delta \in\{0, \pm 1\}$. The normal form theory ensures that $p_{0}$ is orbitally linearizable if, and only if, $\delta=0$. We are assuming that $p_{0}$ is not orbitally linearizable and, thus, $\delta \neq 0$.

Let us consider $\tilde{V}(X, Y)$ to be the formal inverse integrating factor of system (13) constructed with the transformation of $V(x, y)$ with the near-identity normalizing change of variables divided by the Jacobian of the change. We have that $\tilde{V}(X, Y)=$ $X Y \tilde{u}(X, Y)$, where $\tilde{u}$ is a formal series such that $\tilde{u}(0,0) \neq 0$. We can assume, without loss of generality, that $\tilde{u}(0,0)=1$. Easy computations show that if $\tilde{u}$ is constant, we have that $\tilde{V}(X, Y)$ cannot be an inverse integrating factor of system (13) with $\delta \neq 0$. If we have that $\tilde{u}$ is not a constant, we develop $\tilde{u}$ as a formal series in $X$ and $Y$ and we can write $\tilde{u}=1+V_{s}(X, Y)+\cdots$, where the dots correspond to terms of order strictly greater than $s$ in $X$ and $Y$ and $V_{s}(X, Y)$ is a homogeneous polynomial in $X$ and $Y$ of degree $s$. We consider the partial differential equation satisfied by $\tilde{V}$ :

$$
\begin{aligned}
& p X\left[1+\delta\left(U^{\ell}+a U^{2 \ell}\right)\right] \frac{\partial \tilde{V}}{\partial X}-q Y \frac{\partial \tilde{V}}{\partial Y} \\
& \quad=\left(p-q+p \delta(1+\ell q) U^{\ell}+a \delta p(1+2 \ell q) U^{2 \ell}\right) \tilde{V} .
\end{aligned}
$$


We equate terms of the same lowest degree; we deduce that $s=\ell(p+q)$ and that

$$
p X \frac{\partial V_{s}}{\partial X}-q Y \frac{\partial V_{s}}{\partial Y}=\delta \ell p q X^{\ell q} Y^{\ell p}
$$

Easy computations show that the general solution of this partial differential equation is $V_{s}(X, Y)=\delta \ell^{2} p q^{2} U^{\ell} \ln X+G(U)$, where $G$ is an arbitrary smooth function. We deduce that this partial differential equation has no polynomial solution $V_{s}(X, Y)$ unless $\delta=0$.

We conclude that the existence of such an inverse integrating factor $\tilde{V}(X, Y)$ implies that $\delta=0$ in contradiction with our hypothesis.

We observe that homoclinic loops considered in Proposition 11 are simple, since the hyperbolic saddle point is strong. As we have already mentioned in the Introduction, it is well known that its cyclicity is 1 . Therefore, the obstruction to the existence of an analytic inverse integrating factor in a neighborhood of these homoclinic loops is not relevant in the context of bifurcation theory.

The following example provides a realization of the thesis stated in Proposition 11. that is, we illustrate the existence of homoclinic loops where no analytic inverse integrating factor can exist in a neighborhood of it. We consider the system

$$
\dot{x}=-x+2 y+x^{2}, \quad \dot{y}=2 x-y-3 x^{2}+\frac{3}{2} x y
$$

studied in [1]. The origin is a strong saddle because it has eigenvalues $\mu=-3$ and $\lambda=1$, and hence $r=3 \neq 1$. Moreover, system (14) possesses a homoclinic loop $\Gamma$ through the origin contained in the invariant algebraic curve $f(x, y)=$ $x^{2}(1-x)-y^{2}=0$ and having inside a focus. After a linear change of variables, we write system (14) with Jordan linear part as

$$
\dot{x}=-3 x-\frac{11}{8} x^{2}+2 x y-\frac{5}{8} y^{2}, \quad \dot{y}=y-\frac{7}{8} x^{2}+x y-\frac{1}{8} y^{2} .
$$

A computation using the normal form method shows that the above system is conjugated to the system

$$
\dot{x}=-3 x, \dot{y}=y-\frac{86579}{248832} x y^{4},
$$

up to homogeneous degree 15 included. Therefore we conclude that system (14) is not formally orbitally linearizable near the origin. Thus, applying Proposition 11 , there exists no analytic inverse integrating factor $V(x, y)$ for system (14) defined in a neighborhood of $\Gamma$.

We have used the method described in [7] to show that system (14) has no irreducible invariant algebraic curve except $x^{2}(1-x)-y^{2}=0$, nor exponential factors. Therefore, from the Darboux theory of integrability, it can be shown that this system has no Liouvillian first integral. For the definitions and results related to invariant algebraic curves, exponential factors and the Darboux theory of integrability, see [7] and the references therein.

In this paper we will always assume as a hypothesis the existence of an analytic inverse integrating factor $V$ defined on a neighborhood of $\Gamma$. Under this condition, we now refer to the uniqueness problem. In the last section of this work, we present several examples of differential systems with a homoclinic loop which satisfy all our hypotheses and in which an explicit expression of an analytic inverse integrating factor is given. 
Proposition 12. Consider the analytic system (11) having a compact loop $\Gamma$ with a Poincaré return map different from the identity. Then, assuming the existence of an analytic inverse integrating factor $V(x, y)$ of (1) defined in a neighborhood of $\Gamma$, we have that $V(x, y)$ is unique up to a multiplicative constant.

Proof. Assume that we have two different analytic inverse integrating factors $V$ and $\bar{V}$ defined in a neighborhood $\mathcal{N}$ of $\Gamma$. From Theorem 10, it follows that $\Gamma \subset V^{-1}(0)$ and $\Gamma \subset \bar{V}^{-1}(0)$. Let $m$ and $\bar{m}$ be the multiplicities of vanishing of $V$ and $\bar{V}$ on $\Gamma$, respectively. We can assume $m \geq \bar{m}$ and we can construct for system (1) the first integral $H(x, y)=V / \bar{V}$ which is analytic in $\mathcal{N}$. Lemma 7 ensures that this quotient has no poles in $\mathcal{N}$. The existence of $H$ is in contradiction with the fact that $\Gamma$ is an $\alpha$ - or $\omega$-limit set with a return map different from the identity.

As a corollary of the proof of this proposition we have that if there exists an analytic inverse integrating factor defined in a neighborhood of a limit cycle, then it is unique up to a multiplicative constant.

An easy reasoning shows that if we assume the existence of an analytic inverse integrating factor $V(x, y)$ defined in a neighborhood of a compact loop $\Gamma$, through the singular point $p_{0}$ and whose Poincaré return map is not the identity, and if there exists a unique formal inverse integrating factor $\bar{V}(x, y)$ in a neighborhood $D$ of $p_{0}$, then $V(x, y)=\bar{V}(x, y)$ for all $(x, y) \in D$ up to a multiplicative constant.

Let $\Pi(\sigma)$ be the Poincaré return map associated to a homoclinic loop $\Gamma$ and defined over a transversal section $\Sigma$. Then $\Pi$ is the composition $\Pi=R \circ \Delta$, where $R$ is an analytic diffeomorphism defined in a neighborhood of the regular part $\phi(t)$ of $\Gamma$ and $\Delta$ is the transition map defined in a neighborhood of the critical point $p_{0}$. We parameterize $\Sigma$ by a real parameter $\sigma \geq 0$, and $\sigma=0$ corresponds to the base point. In our notation $\sigma$ denotes both a point in $\Sigma$ and the corresponding real value which parameterizes it. Theorem 3 relates the inverse integrating factor and the diffeomorphism defined in a neighborhood of any regular orbit by the flow of system (1). Let us consider a homoclinic orbit as a regular orbit and remark that Proposition 8 also applies in the following sense. In case we consider a homoclinic orbit, the limiting case in which the two sections tend to the saddle point $p_{0}$ with $\Sigma_{1}$ following the unstable separatrix and $\Sigma_{2}$ the stable separatrix gives that this Poincaré map coincides with the aforementioned regular map $R$. We remark that, if $\mathcal{I}=(a, b)$ covers the whole homoclinic orbit in the sense of the flow, this limiting case corresponds to going from $\Sigma_{1}$, based on a value of $s$ such that $s \rightarrow a^{+}$, to $\Sigma_{2}$ based on a value of $s$ such that $s \rightarrow b^{-}$.

We note that, since $V(x, y)$ is a well-defined function in a neighborhood of the whole $\Gamma$, the function $\tilde{V}(s, n)$ takes the same value in the limiting case, i.e., when $s$ tends to the boundaries of $\mathcal{I}$. We define $\tilde{V}(a, n)=\lim _{s \rightarrow a^{+}} \tilde{V}(s, n)$ and $\tilde{V}(b, n)=\lim _{s \rightarrow b^{-}} \tilde{V}(s, n)$ and these limits exist from the same reasoning. Moreover, $\tilde{V}(a, n)=\tilde{V}(b, n)$. Thus, the result stated in Proposition 8 is also valid for studying the regular map $R$ associated to a homoclinic orbit defined with sections in the limiting case.

In order to relate inverse integrating factors and the Poincaré return map associated to a homoclinic loop, we need to study the local behavior of the solutions in a neighborhood of the critical saddle point $p_{0}$. 
The following proposition establishes some relationships between the vanishing multiplicity of $V$ on the separatrices of a hyperbolic saddle point $p_{0}$ and the nature of $p_{0}$, provided that $V\left(p_{0}\right)=0$.

Proposition 13. Let $V(x, y)$ be an analytic inverse integrating factor defined in a neighborhood of a hyperbolic saddle point $p_{0}$ with eigenvalues $\mu<0<\lambda$ of an analytic system (11). Let us consider $f_{\lambda}(x, y)=0$ and $f_{\mu}(x, y)=0$, the local analytic expression of each of the separatrices associated to $p_{0}$, where the subindex denotes the corresponding eigenvalue. Then the following statements hold:

(i) If $p_{0}$ is strong, then $V\left(p_{0}\right)=0$.

(ii) If $p_{0}$ is nonresonant, then $V=f_{\lambda} f_{\mu} u$ with $u\left(p_{0}\right) \neq 0$.

(iii) If $p_{0}$ is $p: q$ resonant and strong, then $V=f_{\lambda}^{1+k p} f_{\mu}^{1+k q} u$ with $u\left(p_{0}\right) \neq 0$ and an integer $k \geq 0$.

(iv) If $p_{0}$ is weak and $V\left(p_{0}\right)=0$, then $V=f_{\lambda}^{m} f_{\mu}^{m} u$ with $u\left(p_{0}\right) \neq 0$ and $m a$ natural number with $m \geq 1$.

Proof. Statement (i) is clear from the definition (2) of an inverse integrating factor.

The other three statements are based on a result due to Seidenberg [23]; see also 7]. Since $V$ is analytic, we can locally factorize near $p_{0}$ as $V=f_{\lambda}^{m_{1}} f_{\mu}^{m_{2}} u$ with $u\left(p_{0}\right) \neq 0$ and $m_{i}$ nonnegative integers. Then, we have $\operatorname{div} \mathcal{X}\left(p_{0}\right)=m_{1} \mu+m_{2} \lambda$. Hence, $\left(m_{1}-1\right) \mu+\left(m_{2}-1\right) \lambda=0$ and statements (ii)-(iv) easily follow.

By an affine change of coordinates, in a neighborhood of a hyperbolic saddle, system (11) can be written as $\dot{x}=\lambda x+f(x, y), \dot{y}=\mu y+g(x, y)$, where $f$ and $g$ are analytic in a neighborhood of the origin with lowest terms at least of second order and $\mu<0<\lambda$. This hyperbolic saddle is analytically orbitally linearizable if there exists an analytic near-identity change of coordinates transforming the system to $\dot{x}=\lambda x h(x, y), \dot{y}=\mu y h(x, y)$ with $h(0,0)=1$. In case $\lambda+\mu=0$, then $V(x, y)=x^{k} y^{k} h(x, y)$ is a 1 -parameter family of analytic inverse integrating factors of the former system for any integer $k \geq 1$.

Let us consider again an analytic system (1) where $p_{0}$ is a weak hyperbolic saddle, that is, $\lambda+\mu=0$. After the above affine change of coordinates and rescaling the time, if necessary, the system can be written as

$$
\dot{x}=x+f(x, y), \dot{y}=-y+g(x, y),
$$

where $f$ and $g$ are the nonlinear terms. The existence is well known of a formal near-identity change of coordinates $(x, y) \mapsto(X, Y)=(x+\cdots, y+\cdots)$ that brings system (15) into the Poincaré normal form

$$
\dot{X}=X\left[1+\sum_{i \geq 1} a_{i}(X Y)^{i}\right], \dot{Y}=-Y\left[1+\sum_{i \geq 1} b_{i}(X Y)^{i}\right]
$$

From this expression, we see that system (15) has an analytic first integral in a neighborhood of the saddle if and only if the saddle quantities $\alpha_{i+1}:=a_{i}-b_{i}$ are zero for all $i \geq 1$; see [19] and the references therein for a review. In particular we observe that system (15) has a 1:1 resonant saddle at the origin. In [15], it is proved that a planar dynamical system is analytically orbitally linearizable at a resonant hyperbolic saddle, that is, whose hyperbolicity ratio $r$ is a rational number, if and only if it has an analytic first integral in a neighborhood of the saddle. 
In 4], Brjuno shows that any resonant hyperbolic saddle point of an analytic system is analytically orbitally linearizable if and only if it is formally orbitally linearizable. In particular, this fact means that either there exists at least one saddle quantity $\alpha_{i}$ different from zero or the system becomes analytically orbitally linearizable. Moreover, we can prove the following result.

Theorem 14. Let us consider an analytic system (10) with a hyperbolic weak saddle point $p_{0}$ whose separatrices are locally given by $f_{1}(x, y)=0$ and $f_{-1}(x, y)=0$. Let $V=f_{1}^{m} f_{-1}^{m} u$ be an analytic inverse integrating factor defined in a neighborhood of $p_{0}$ such that $u\left(p_{0}\right) \neq 0$ and $m$ is a nonnegative integer. If $p_{0}$ is not analytically orbitally linearizable, then $m \geq 2$ and the first nonvanishing saddle quantity is $\alpha_{m}$.

Proof. We can always assume that system (11) takes the form (15) where the hyperbolic weak saddle point $p_{0}$ is at the origin and the expression of the separatrices takes the form $f_{1}(x, y)=y+o(x, y), f_{-1}(x, y)=x+o(x, y)$. As a result of Proposition 13 the analytic inverse integrating factor reads $V(x, y)=f_{1}^{m} f_{-1}^{m} u$ with nonnegative integer $m \geq 0$ and $u(0,0) \neq 0$.

If $m=0$, then $V\left(p_{0}\right) \neq 0$ and there exists an analytic first integral defined on a neighborhood of $p_{0}$ for system (11). Hence, $p_{0}$ is analytically orbitally linearizable and the saddle quantities of $p_{0}$ satisfy $\alpha_{i}=0$ for any $i \geq 1$.

If $m=1$, then $V=f_{1} f_{-1} u$ with $u\left(p_{0}\right) \neq 0$. Then, by statement (iii) of Theorem 5.10 of 9 , we conclude that $p_{0}$ is also analytically orbitally linearizable and the saddle quantities of $p_{0}$ satisfy $\alpha_{i}=0$ for any $i \geq 1$.

If $m \geq 2$, we are going to prove that the saddle quantities at the origin satisfy $\alpha_{i}=0$ for $i=1,2, \ldots, m-1$ and that $\alpha_{m} \neq 0$. We do a formal near-identity change of coordinates $(x, y) \mapsto(X, Y)$ transforming system (15) into the Poincaré normal form (16). In these normalizing coordinates, system (16) has the formal inverse integrating factor

$$
\bar{V}(X, Y)=X Y \sum_{i \geq 1} \alpha_{i+1}(X Y)^{i} .
$$

We observe that functions which define system (16) give $\bar{V}(X, Y)=\dot{X} / X+\dot{Y} / Y$. We remark that $\bar{V}(X, Y)$ is a univariate function of the variable $X Y$. Since there is at least one nonzero saddle quantity, system (16) has no formal first integral near the origin. In particular, (17) is the unique (up to multiplicative constants) formal inverse integrating factor of system (16). We note that performing the above formal near-identity change of coordinates to the inverse integrating factor $V(x, y)$, we get a formal inverse integrating factor of system (16) of the form $X^{m} Y^{m} \bar{u}(X, Y)$, with $\bar{u}(0,0) \neq 0$. Therefore, from uniqueness, we must have that it coincides with the expression (17). Thus, we have $\alpha_{i}=0$ for $i=1,2, \ldots, m-1$ and that $\alpha_{m} \neq 0$.

We have analyzed the behavior of the flow near a hyperbolic saddle point $p_{0}$ when an analytic inverse integrating factor $V$ is known. In particular, we shall see that the transition map $\Delta$ near $p_{0}$ can be studied by the vanishing multiplicity of $V$ in the separatrices of $p_{0}$. Let us consider two transversal sections $\Sigma_{2}$ and $\Sigma_{1}$ in a sufficiently small neighborhood of $p_{0}$, where $\Sigma_{2}\left(\right.$ resp. $\left.\Sigma_{1}\right)$ is based on a point over the stable (resp. unstable) separatrix of $p_{0}$. We parameterize $\Sigma_{2}$ by a real parameter $\sigma \geq 0$, and $\sigma=0$ corresponds to the base point. We recall that the transition map $\Delta: \Sigma_{2} \rightarrow \Sigma_{1}$ is defined as $\Delta(\sigma)=\Phi(\tau(\sigma) ; \sigma) \cap \Sigma_{1}$, where $\Phi(t ; \sigma)$ is the flow associated to system (1) with initial condition the point $\sigma \in \Sigma_{2}$, and 
$\tau(\sigma)$ is the minimal positive time such that the trajectory through $\sigma$ intersects $\Sigma_{1}$. The explicit asymptotic expansion of $\Delta(\sigma)$ was given by Dulac [10] in terms of the hyperbolicity ratio $r$ and saddle quantities $\alpha_{i}$ of $p_{0}$ as follows:

$$
\Delta(\sigma)= \begin{cases}c \sigma^{r}(1+o(1)), c>0 & \text { if } \quad r \neq 1, \\ \sigma+\alpha_{k} \sigma^{k} \ln \sigma+o\left(\sigma^{k} \ln \sigma\right) & \text { if } \quad \alpha_{1}=\cdots=\alpha_{k-1}=0, \alpha_{k} \neq 0, \\ \sigma & \text { if } \quad \alpha_{i}=0, \text { for all } i \geq 1 .\end{cases}
$$

We remark that the identity $\Delta(\sigma)=\sigma$ in the latter case is understood in the formal sense.

Since the Poincaré return map $\Pi(\sigma)$ associated to the compact homoclinic loop $\Gamma$ is the composition $\Pi=R \circ \Delta$ and is different from the identity, we only have the four possibilities (i)-(iv) described in Theorem 9 .

In the following theorem, the cyclicity of $\Gamma$ denotes the maximum number of limit cycles that bifurcate from $\Gamma$ under smooth perturbations of (11).

Theorem 15. Let $\Gamma$ be a compact homoclinic loop through the hyperbolic saddle point $p_{0}$ of system (11) whose Poincaré return map is not the identity. Let $V$ be an analytic inverse integrating factor defined in a neighborhood of $\Gamma$ with vanishing multiplicity $m$ over $\Gamma$. Then the following statements hold:

(a) $m \geq 1$.

(b) If $p_{0}$ is strong, then $m=1$ and the cyclicity of $\Gamma$ is 1 .

(c) If $p_{0}$ is weak, then:

(c.1) If $p_{0}$ is not analytically orbitally linearizable, then $m \geq 2, \alpha_{i}=\beta_{i}=0$ for $i=1,2, \ldots, m-1$ and $\alpha_{m} \neq 0$. Moreover, the cyclicity of $\Gamma$ is $2 m-1$.

(c.2) If $p_{0}$ is analytically orbitally linearizable, then $\beta_{1}=\beta_{2}=\cdots=\beta_{m-1}=$ 0 and $\beta_{m} \neq 0$. Moreover, the cyclicity of $\Gamma$ is $2 m$.

Proof. Statement (a) is a straight consequence of the fact that $\Gamma \subset V^{-1}(0)$; see Theorem 10.

Statement (b) follows by taking into account statements (ii) and (iii) of Proposition 13. where we recall that the vanishing multiplicity of $V$ in each of the separatrices of $p_{0}$ needs to be the same since $\Gamma$ is a loop. In particular, we observe that the value of $k$ in statement (iii) of Proposition 13 must be zero by the same argument.

We assume that $p_{0}$ is weak. We may have that all its associated saddle quantities are zero or that there is at least one saddle quantity different from zero. If this last case applies, the first nonvanishing saddle quantity is $\alpha_{m}$ as a result of Theorem 14. Therefore, we compute the $m^{\text {th }}$ saddle quantity associated to $p_{0}$, knowing that the previous saddle quantities need to be zero and we can determine if $p_{0}$ is analytically orbitally linearizable (if $\alpha_{m}=0$ ) or not (if $\alpha_{m} \neq 0$ ). The case (c.1) in the theorem corresponds to $\alpha_{m} \neq 0$ and the case (c.2) to $\alpha_{m}=0$.

The case (c.1) is a consequence of Theorem 14 and part (b) of Proposition 8. More concretely, since $p_{0}$ is not analytically orbitally linearizable, then using Theorem 14 we have $m \geq 2, \alpha_{i}=0$ for $i=1,2, \ldots, m-1$ and $\alpha_{m} \neq 0$. In addition, by part (b) of Proposition 8 , we get $\beta_{i}=0$ for $i=1,2, \ldots, m-1$. We remark that, in this case, either $\beta_{m} \neq 0$ or $\beta_{k}=0$ for all $k \geq 1$. In any case, from statement (iii) of Theorem 9, the cyclicity of $\Gamma$ is $2 m-1$.

Finally, the proof of (c.2) works as follows. Since $p_{0}$ is analytically orbitally linearizable, then $\alpha_{k}=0$ for all $k \geq 1$. By hypothesis, the Poincaré map is not the 
identity, so $\beta_{k}=0$ for all $k \geq 1$ is not possible. Hence, by part (b) of Proposition 8, we get that $\beta_{i}=0$ for $i=1,2, \ldots, m-1$ and $\beta_{m} \neq 0$. Thus, using statement (iv) of Theorem 9, the cyclicity of $\Gamma$ is $2 m$.

Proof of Theorem [6. The thesis of Theorem 6 is a corollary of Theorem 15.

\section{EXAmples}

This section contains several examples which illustrate and complete our results. The first example shows how to give an implicit expression of the Poincaré map associated to a regular orbit via identity (7). The second example is given to show the existence of analytic planar differential systems with a compact homoclinic loop $\Gamma$ whose cyclicity can be determined by the vanishing multiplicity of an inverse integrating factor defined in a neighborhood of it. The third example targets showing that there is no upper bound for the number of limit cycles which bifurcate from a compact homoclinic loop whose Poincaré return map is the identity. The system considered in this example has a denumerable family of inverse integrating factors such that for any natural number $n$ there exists an analytic inverse integrating factor whose vanishing multiplicity on the compact homoclinic loop is $n$.

Example 1. The following system is studied in [5] where a complete description of its phase portrait in terms of the parameters is given using the inverse integrating factor as a key tool. The system

$$
\begin{aligned}
& \dot{x}=\lambda x-y+\lambda m_{1} x^{3}+\left(m_{2}-m_{1}+m_{1} m_{2}\right) x^{2} y+\lambda m_{1} m_{2} x y^{2}+m_{2} y^{3}, \\
& \dot{y}=x+\lambda y-x^{3}+\lambda m_{1} x^{2} y+\left(m_{1} m_{2}-m_{1}-1\right) x y^{2}+\lambda m_{1} m_{2} y^{3},
\end{aligned}
$$

where $\lambda, m_{1}$ and $m_{2}$ are arbitrary real parameters, has the following inverse integrating factor

$$
V(x, y)=\left(x^{2}+y^{2}\right)\left(1+m_{1} x^{2}+m_{1} m_{2} y^{2}\right) .
$$

In [5] it is shown that if $\lambda \neq 0, m_{1}<0, m_{1} \neq-1$ and $m_{2}>0$, then the ellipse defined by $1+m_{1} x^{2}+m_{1} m_{2} y^{2}=0$, and which we denote by $\gamma$, is a hyperbolic limit cycle of system (18). Moreover, with the described values of the parameters, we have that the origin of coordinates is a strong focus whose boundary of the focal region is $\gamma$. Moreover, since the vanishing set of $V$ is only the focus at the origin and the limit cycle $\gamma$, we deduce by Theorem 10 that the region, in the outside of $\gamma$, in which the orbits spiral towards or backwards to it, is unbounded.

We remark that we reencounter that this limit cycle is hyperbolic using that the vanishing multiplicity of the inverse integrating factor over it is 1 . In this example we are not concerned with the multiplicity of the limit cycle but with the Poincaré return map associated to it. We are going to use the ordinary differential equation stated in (7) to give an implicit expression for the Poincaré map associated to $\gamma$ in system (18). We can parameterize the ellipse by $\left(\sqrt{m_{2}} \cos (s), \sin (s)\right) / \sqrt{-m_{1} m_{2}}$ with $s \in[0,2 \pi)$ and we perform the corresponding change to curvilinear coordinates: $(x, y) \mapsto(s, n)$ with $x=\left(\sqrt{m_{2}}-n\right) \cos (s) / \sqrt{-m_{1} m_{2}}$ and $y=$ $\left(1-\sqrt{m_{2}} n\right) \sin (s) / \sqrt{-m_{1} m_{2}}$. We obtain an ordinary differential equation of the form (5) which describes the behavior of the solutions of system (18) in a neighborhood of the limit cycle (for $n=0$ ). The corresponding inverse integrating factor 
$\tilde{V}(s, n)$ is a $2 \pi$-periodic function in $s$ and it satisfies:

$$
\tilde{V}(0, n)=\frac{m_{1} n\left(n-\sqrt{m_{2}}\right)\left(n-2 \sqrt{m_{2}}\right)}{n^{2}-2 n \sqrt{m_{2}}+m_{2}+m_{1} m_{2}} .
$$

The ordinary differential equation (7) reads $\tilde{V}(2 \pi, \Pi(n))=\tilde{V}(0, n) \Pi^{\prime}(n)$ for this system, where $n$ can be taken as a real parameter of the section $\Sigma:=\{s=0\}$ and such that $n=0$ corresponds to the limit cycle. We observe that the point $n=$ $\sqrt{m_{2}}$ corresponds to the focus point at the origin of system (18). This differential equation can be written in the following Pfaffian form:

$$
\frac{d n}{\tilde{V}(0, n)}=\frac{d \Pi}{\tilde{V}(2 \pi, \Pi)},
$$

whose integration gives

$$
\frac{\left(\Pi(n)\left(\Pi(n)-2 \sqrt{m_{2}}\right)\right)^{\left(1+m_{1}\right) / 2}}{\left(\Pi(n)-\sqrt{m_{2}}\right)^{m_{1}}}=k_{0} \frac{\left(n\left(n-2 \sqrt{m_{2}}\right)\right)^{\left(1+m_{1}\right) / 2}}{\left(n-\sqrt{m_{2}}\right)^{m_{1}}},
$$

where $k_{0}$ is an arbitrary constant of integration. We remark that if $k_{0}=1$, then the function $\Pi(n):=n$ satisfies the implicit identity (20).

We observe that the integration of (7) always gives rise to a Pfaffian form of separated variables. Moreover, in the case that a return is involved we have that this Pfaffian form is symmetric in $\Pi$ and $n$, because the function $\tilde{V}(s, n)$ needs to be $L$-periodic in $s$.

We are interested in the Poincaré map associated to the limit cycle $\gamma$. Since it is a hyperbolic limit cycle, we have that $\Pi(n)=e^{\beta_{1}} n+\mathcal{O}\left(n^{2}\right)$ with $\beta_{1} \neq 0$, as described in Proposition 8. Using this expression and identity (20), we deduce that $k_{0}=\exp \left(\beta_{1}\left(m_{1}+1\right) / 2\right)$. Moreover, it can be shown that $\beta_{1}=-2 \lambda T$, where $T$ is the minimal positive period of $\gamma$ and takes the value $T=2 \pi m_{1} /\left(1+m_{1}\right)$ if $m_{1}<-1$ and $T=-2 \pi m_{1} /\left(1+m_{1}\right)$ if $-1<m_{1}<0$. Therefore, $k_{0}=e^{-2 \lambda m_{1} \pi}$ if $m_{1}<-1$ and $k_{0}=e^{2 \lambda m_{1} \pi}$ if $-1<m_{1}<0$. We note that the inverse integrating factor determines the multiplicity of the limit cycle $\gamma$ but not its stability.

Example 2. We fix an integer number $m$ with $m \geq 1$ and we consider the algebraic curve $f=0$ with $f(x, y)=y^{2}-(1-x)^{2}(1+x)$. We have that $f=0$ has an oval in the range $-1 \leq x \leq 1$ with a double point in $(1,0)$. We denote this oval by $\Gamma$. Let us consider the planar differential system:

$$
\begin{gathered}
\dot{x}=-\left(\left[(1-m) g(x, y)+f(x, y)^{m-1}\right] \frac{\partial f}{\partial y}+f(x, y) \frac{\partial g}{\partial y}\right)\left(x^{2}+y^{2}\right) q(x, y) \\
-f(x, y)^{m}\left(2(x+y) q(x, y)+\left(x^{2}+y^{2}\right) \frac{\partial q}{\partial y}\right), \\
\dot{y}=\left(\left[(1-m) g(x, y)+f(x, y)^{m-1}\right] \frac{\partial f}{\partial x}+f(x, y) \frac{\partial g}{\partial x}\right)\left(x^{2}+y^{2}\right) q(x, y) \\
+f(x, y)^{m}\left(2(x-y) q(x, y)+\left(x^{2}+y^{2}\right) \frac{\partial q}{\partial x}\right),
\end{gathered}
$$

where $g(x, y)$ and $q(x, y)$ are polynomials such that the algebraic curve $g=0$ does not intersect the oval $\Gamma$ and the algebraic curve $q=0$ does not contain any point in the closed region bounded by $\Gamma$. This system has $\Gamma$ as homoclinic loop, where the critical point $(1,0)$ is a hyperbolic weak saddle point. Moreover, the origin 
of this system is a strong focus. It can be shown that the function $V(x, y)=$ $\left(x^{2}+y^{2}\right) f(x, y)^{m} q(x, y)$ is an inverse integrating factor, which is analytic in the whole affine plane. Since the origin is a strong focus and $V$ is analytic in $\mathbb{R}^{2}$, we have by Theorem 10 that the boundary of the focal region must be contained in the zero-set of $V$. We conclude that the boundary of this focal region needs to be $\Gamma$ and, therefore, we have that $\Gamma$ is a compact homoclinic loop through a hyperbolic saddle and whose associated Poincaré return map is not the identity. Moreover, we deduce that the vanishing multiplicity of $V$ over $\Gamma$ is $m$. The following function,

$$
H(x, y)=(x+i y)^{1-i}(x-i y)^{1+i} f(x, y) e^{g(x, y) / f(x, y)^{m-1}} q(x, y),
$$

is a first integral of system (21). In the work 20] it is shown that this form of a first integral implies that the inverse integrating factor is polynomial and, thus, well defined over the entire real plane. When $m=1, H(x, y)$ provides an analytic first integral defined in a neighborhood of the saddle point $(1,0)$ and we deduce that the saddle quantities associated to the critical point $(1,0)$ are all zero. Thus, using Theorem 15, we conclude that when $m=1$ the cyclicity of $\Gamma$ in system (21) is 2 .

When $m>1$, using Theorem [15, we deduce that the cyclicity of $\Gamma$ in system (21) is $2 m-1$ if $\alpha_{m} \neq 0$ and $2 m$ if $\alpha_{m}=0$.

Example 3. Let us consider the following planar differential system:

$$
\dot{x}=-2 y, \quad \dot{y}=-2 x+3 x^{2},
$$

which has a homoclinic loop $\Gamma$ contained in the invariant algebraic curve $f=0$ with $f(x, y):=y^{2}-x^{2}+x^{3}$. Since the origin $(0,0)$ is the saddle point contained in $\Gamma$ and it is a hyperbolic saddle point, we have that $\Gamma$ has associated a Poincaré return map, which is the identity as the system is Hamiltonian with $H=f$. In addition to the hyperbolic saddle point at the origin, the system possesses a singular point of center type in the point with coordinates $(2 / 3,0)$ and no other critical point in the affine plane. We note that any function of the form $V=f(x, y)^{n}$, with $n$ a natural number, provides an analytic inverse integrating factor for the system in the whole plane $\mathbb{R}^{2}$. Thus, we cannot define the vanishing multiplicity of an inverse integrating factor on $\Gamma$.

We observe that for any natural number $n$, there exist perturbations of system (22) with at least $n$ limit cycles which bifurcate from the considered homoclinic loop $\Gamma$, that is, there is no finite upper bound for the cyclicity of $\Gamma$. In the following paragraph, we illustrate this fact by exhibiting a suitable perturbation. We remark that the fact that the vanishing multiplicity of an inverse integrating factor on $\Gamma$ is not defined is coherent with the nonexistence of an upper bound for the cyclicity of $\Gamma$.

For instance, let us fix a natural value $n$ and let $\varepsilon$ be a nonzero real number with $|\varepsilon|$ small enough. We take $a_{i} \in \mathbb{R}, i=1,2, \ldots, n$, such that $a_{i} \neq a_{j}$ if $i \neq j$ and with $0<a_{i} \varepsilon<4 / 27$. For each $i$ we have that the algebraic curve $f_{i}=0$ with $f_{i}:=f+a_{i} \varepsilon$ has an oval with the point $(2 / 3,0)$ in its inner region and the point $(0,0)$ in its outer region. Moreover, if $a_{i} \varepsilon<a_{j} \varepsilon$, the oval defined by $f_{i}=0$ contains the oval $f_{j}=0$ in its inner region. The following system,

$$
\dot{x}=-2 y, \quad \dot{y}=-2 x+3 x^{2}+\varepsilon y \prod_{i=1}^{n}\left(f+a_{i} \varepsilon\right),
$$


is a perturbation of system (22) and exhibits each one of the ovals defined by the curves $f_{i}=0$ as periodic orbits. Easy computations show that, if $|\varepsilon|>0$ is small enough, the only critical points of the perturbed system are $(0,0)$, which is a hyperbolic saddle point, and $(2 / 3,0)$, which is a strong focus. It is also easy to check that each oval described by $f_{i}=0$ is a hyperbolic limit cycle of the perturbed system which bifurcates from $\Gamma$. We have, in this way, that the considered perturbed system has at least $n$ limit cycles which bifurcate from $\Gamma$.

\section{ACKNOWLEDGEMENTS}

We would like to thank Professor Cristóbal García from Universidad de Huelva (Spain) for his commentaries on normal form theory and the corresponding computations done for system (14). We also thank the referee for very useful remarks.

\section{REFERENCES}

[1] A.A. Andronov, E. A Leontovich, I. I. Gordon, And A. G. Mă̌er, Theory of bifurcations of dynamic systems on a plane, John Wiley and Sons, New York, 1973. MR0344606 (49:9345)

[2] V.I. Arnold And Y.S. IL'yashenko, Encyclopedia of Math. Sci. Vol. 1 [Dynamical Systems, 1], Springer-Verlag, Berlin, 1988. MR970794

[3] L.R. Berrone and H. Giacomini, On the vanishing set of inverse integrating factors, Qual. Th. Dyn. Systems 1 (2000), 211-230. MR1808363(2001k:37029)

[4] A.D. Bruuno, Analytic form of differential equations, Trans. Moscow Math. Soc. 25 (1971), 131-288. MR0377192 (51:13365)

[5] J. Chavarriga, H. Giacomini and J. Giné, On a new type of bifurcation of limit cycles for a planar cubic system. Nonlinear Anal. 36 (1999), Ser. A: Theory Methods, 139-149. MR1668868 (2000a:34056)

[6] J. Chavarriga, H. Giacomini, J. Giné and J. Llibre, On the integrability of twodimensional flows, J. Diff. Equations 157 (1999), 163-182. MR.1710019 (2000h:37058)

[7] J. Chavarriga, H. Giacomini and M. Grau, Necessary conditions for the existence of invariant algebraic curves for planar polynomial systems, Bull. Sci. Math. 129 (2005), 99126. MR2123262 (2005k:34123)

[8] L.A. Cherkas, Structure of a succesor function in the neighborhood of a separatrix of a perturbed analytic autonomous system in the plane, Translated from Differentsial'nye Uravneniya 17 (1981), 469-478. MR610508 (82i:34029)

[9] C. Christopher, P. Mardĕsić and C. Rousseau, Normalizable, integrable and linearizable saddle points in complex quadratic systems in $\mathbb{C}^{2}$, J. Dynam. Control Systems 9 (2003), 311363. MR.1990240 (2004d:37072)

[10] H. Dulac, Recherche sur les points singuliers des équations différentielles, J. Ecole Polytechnique 2 (1904), 1-25.

[11] I.A. García AND D.S. Shafer, Integral invariants and limit sets of planar vector fields, J. Differential Equations 217 (2005), 363-376. MR2168828 (2006g:34073)

[12] A. Gasull, J. Giné And M. Grau, Multiplicity of limit cycles and analytic $m$-solutions for planar differential systems, J. Differential Equations 240 (2007), 375-398. MR 2351182 (2008g:34079)

[13] H. Giacomini, J. Llibre and M. Viano, On the nonexistence, existence, and uniqueness of limit cycles, Nonlinearity 9 (1996), 501-516. MR1384489 (97a:34073)

[14] H. Giacomini, J. Llibre and M. Viano, Semistable limit cycles that bifurcate from centers, Internat. J. Bifur. Chaos Appl. Sci. Engrg. 13 (2003), 3489-3498. MR.2031154 (2005a:37082)

[15] M. HAN AND K. JiAng, Normal forms of integrable systems at a resonant saddle, Ann. Differential Equations 14 (1998), 150-155. MR1634022 (99g:34079)

[16] M. HAN AND Z. HuAipIng, The loop quantities and bifurcations of homoclinic loops, J. Differential Equations 234 (2007), 339-359. MR2300659 (2008a:34108)

[17] M.A. Jebrane and A. Mourtada, Cyclicité finie des lacets doubles non triviaux. (French) [Finite cyclicity of nontrivial double loops] Nonlinearity 7 (1994), 1349-1365. MR 1294547 (95h:58110) 
[18] A. Jebrane, P. Mardešić And M. Pelletier, A generalization of Francoise's algorithm for calculating higher order Melnikov functions. Bull. Sci. Math. 126 (2002), 705-732. MR:1941082(2004c:34088)

[19] J. LI, Hilbert's 16th problem and bifurcations of planar polynomial vector fields, International Journal of Bifurcation and Chaos 13 (2001), 47-106. MR1965270 (2003k:34002)

[20] J. Llibre and C. Pantazi, Polynomial differential systems having a given Darbouxian first integral, Bull. Sci. Math. 128 (2004), 775-788. MR2099106 (2005m:34070)

[21] R. Roussarie, On the number of limit cycles which appear by perturbation of separatrix loop of planar vector fields, Bol. Soc. Bras. Mat. 17 (1986), 67-101. MR901596 (88i:34061)

[22] R. Roussarie, Bifurcations of planar vector fields and Hilbert's sixteenth problem, Progress in Mathematics Vol 164, Birkhäuser Verlag, 1998. MR1094374 (91j:58004)

[23] A. Seidenberg, Reduction of singularities of the differential equation $A d y=B d x$, Amer. J. Math. 90 (1968) 248-269. MR0220710 (36:3762)

Departament de Matemàtica, Universitat de Lleida, Avda. Jaume II, 69. 25001 Lleida, SPAIN

E-mail address: garcia@matematica.udl.cat

Laboratoire de Mathématiques et Physique Théorique, C.N.R.S. UMR 6083, Faculté des Sciences et Techniques, Université de Tours, Parc de Grandmont 37200 Tours, FRANCE

E-mail address: Hector.Giacomini@lmpt.univ-tours.fr

Departament de Matemàtica, Universitat de Lleida, Avda. Jaume II, 69. 25001 Lleida, SPAIN

E-mail address: mtgrau@matematica.udl.cat 